\title{
CONJOINT ANALYSIS: A NEW METHOD OF ELICITING ENVIRONMENTAL PREFERENCES AND STAKEHOLDER GROUP SEGMENTATION
}

\author{
Stina Alriksson \\ University of Kalmar, Sweden
}

\begin{abstract}
Deciding on an environmental strategy is an important and also difficult task for a manufacturing organisation in Sweden today. Therefore, there is a need for a method to enable organisations to sort and prioritize the environmental objectives and concerns. The Swedish Steel Producers' Association,"Jernkontoret", has initiated a research programme called "Towards a closed steel eco-cycle". The aim is to make the steel eco-cycle sustainable through increased scrap recycling and efficiency. In order to assess what environmental preferences the different stakeholder groups have, an interdisciplinary research project, using conjoint analysis, has been performed where preferences for some of the most important environmental concerns within the steel industry were investigated. Environmental preferences within six stakeholder groups - members of public, representatives from the steel industry, customers to the steel industry, interest groups, decision makers and environmental experts - were evaluated through a conjoint analysis combined with a cluster analysis. The survey was distributed through a questionnaire and the respondents were asked to rank 8 alternatives in a fractional factorial design.
\end{abstract}

The results indicated that emission of carbon dioxide was prioritized over use of nonrenewable energy, use of non-renewable resources and weight reduction of products. A cluster analysis was made on the respondents and eight segments were found, however, this segmentation was markedly different than expected in that the original six groups were not recovered.

The results were communicated back to the participants directly after they responded to the questionnaire and the result was discussed in the group. The reactions from the discussion were documented in order to be made useful in the product development process as well as in the decision process within the steel industry.

\section{KEYWORDS}

Cluster analysis; Conjoint analysis; Experimental design; MLR; PLSR; Steel ecocycle.

\section{INTRODUCTION}

The Swedish Steel Producers' Association (Jernkontoret) has identified a number of sustainability problems for the trade; emission of carbon dioxide, consumption of non-renewable resources such as ore, minerals and fossil fuels, consumption of non-renewable energy, scrap metal that is not retumed, loss of alloys and contamination of scrap just to mention a few. To resolve some of these issues a research programme called "Towards a closed steel eco-cycle" has been initiated (Figure 
1). The aim of the research programme is to make the steel eco-cycle sustainable through a series of concurrent research projects, covering increased scrap recycling and efficiency among others. Lack of knowledge of how stakeholder groups value environmental concerns has been made one of the issues in the research programme, as well as the need of a decision tool in order to make better decisions on environmental issues and objectives. These research issues has been assembled in a research project called "Conjoint analysis as a decision tool".

The last decade, conjoint analysis, and related methods, has been used to assess preferences for environmental issues [1] and the method looks very promising in order to assess stakeholder groups preferences on environmental objectives. In the environmental area, most conjoint studies with an environmental emphasis have covered recreation, hunting, product development and ecosystem management. Using the method to assess environmental concerns covering an entire trade has not been attempted before.

In conjoint analysis the researcher uses experimental planning in order to create a number of hypothetical situations/products were the levels of the attributes are varied. The respondents are presented with the alternate situations/products and asked to rate/rank or choose between the alternatives. Conjoint analysis is a decompositional method where the choice of the respondent is assumed to represent a total sum of "utility" for the alternative. The utility of each attribute can then be estimated by decomposing the total utility [2].

Most conjoint studies use the methods of regression for the analysis of the data. In later years, algorithms have been developed for some of the different special interests of research, e.g. Multi Nomial Logit and Multi Nomial Probit. Yet, another example is the development of the random utility theory (RUT), where comparisons of monetary estimations are made possible between different studies [3].

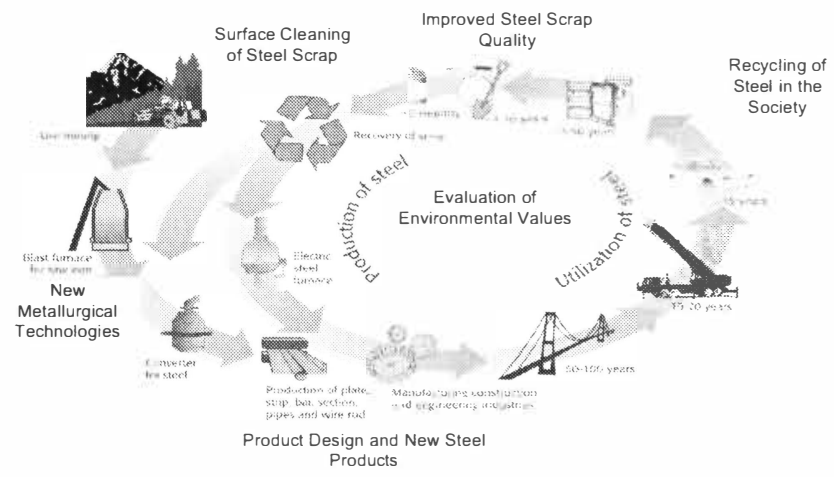

Figure 1. The Steel Eco-cycle research programme. 


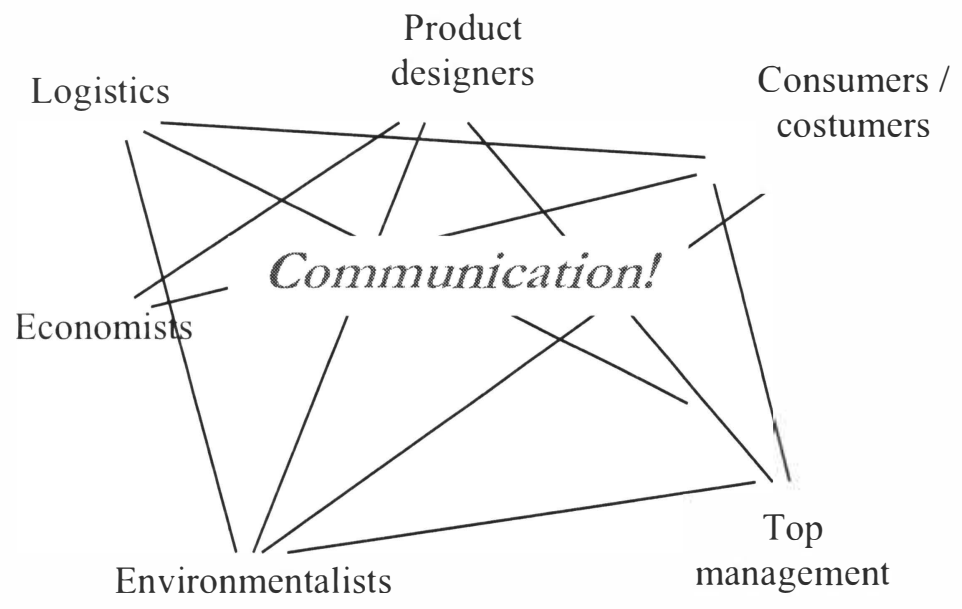

Figure 2. stakeholder group communication leading to eco-efficient decisions and product development.

One of the challenges to address the sustainability problems of the Swedish steel trade is the lack of knowledge on how different stakeholder groups value environmental issues. If this lack of knowledge could be filled it would enable more efficient decisions by the management in the steel companies, concerning environmental objectives. Thus, stakeholder group preferences are essential in order for the management to be able to make correct decisions. Also, if the knowledge that each stakeholder group possesses were used in the decision process, many unnecessary environmental aspects could be avoided.

In this study we have adopted conjoint analysis to address the valuation of some of the major sustainability problems of the steel trade. The main research questions was to find out which of the "Steel eco-cycle" environmental objectives do the different stakeholder groups prefer and if there are any other environmental issues that the respondents find as important or even more important.

Furthermore, we have tried a new way of presenting the results of the conjoint study for the respondents directly after they finished their questionnaires. By presenting the results back to the respondents, a discussion has been initialised where the stakeholder groups are given an opportunity to learn from each other (see Figure 2).

\section{METHOD}

\subsection{Pilot study}

Before the main study was conducted, a pilot study [4] was performed where conjoint analysis was tested on some of the sustainability problems of the steel industry. The following research questions were in focus in the pilot study: 
- Is the method of conjoint analysis applicable on the sustainability problems of an entire trade such as the Swedish steel trade?

- Can multivariate data analysis further enhance the use of the method by providing a way of displaying the results on an individual level?

- Is it possible to extract the preferences of different stakeholder groups from the results?

The pilot study clarified that conjoint analysis works well even for environmental issues for an entire trade, that partial least squares regression enables presentation of results on an individual level. Conjoint analysis combined with cluster analysis makes it possible to find segmentation among the respondents that is not automatically identified. Experiences from the pilot study influenced the design of this study.

\subsection{Conjoint study of environmental objectives of the Steel eco-cycle}

In the study presented here, the four environmental objectives of the research programme "Towards a closed steel eco-cycle" were chosen to represent the major sustainability problems of the trade.

The factors were:

- Use of non-renewable energy,

- Use of non-renewable resources,

- Emissions of carbon dioxide and

- Weight reduction of steel products (high strength steel)

Each attribute was presented in two levels. If all levels of all factors were to be used in the experimental design, the respondents would have had to evaluate 16 alternatives. However, by using a fractional factorial design, eight alternatives were finally used. The alternatives were presented in a table where the respondents were asked to rank and rate the alternatives.

One objection to the choice of attributes in this study may be that they interact, since reduction of the use of non-renewable energy will lead to reduction in emission of carbon dioxide. The same objection can be made for weight reduction of products, where use of nonrenewable resources will decrease at the same time. However, studies has shown that respondents are not affected by unrealistic combinations of attributes and levels [5]

Six groups of respondents were chosen; representatives from the steel industry, customers to the steel industry, steel research institutes, members of the public, decision makers and environmental experts. The questionnaire consisted of three parts, first two pages of information on the attributes (use of non-renewable energy, use of non-renewable resources, emissions of carbon dioxide and weight reduction of products), next, the conjoint task and finally, some socio-economic questions concerning for example gender, marital status, environmental commitment and occupation.

The questionnaire was distributed in two ways; to four of the groups (members of public, decision makers, environmental experts and steel research institutes) the questionnaire was sent by post with an enclosed pre-franked response-envelope, while the steel industries and the steel costumers were visited and the research project was presented orally before the respondents were asked to participate. 


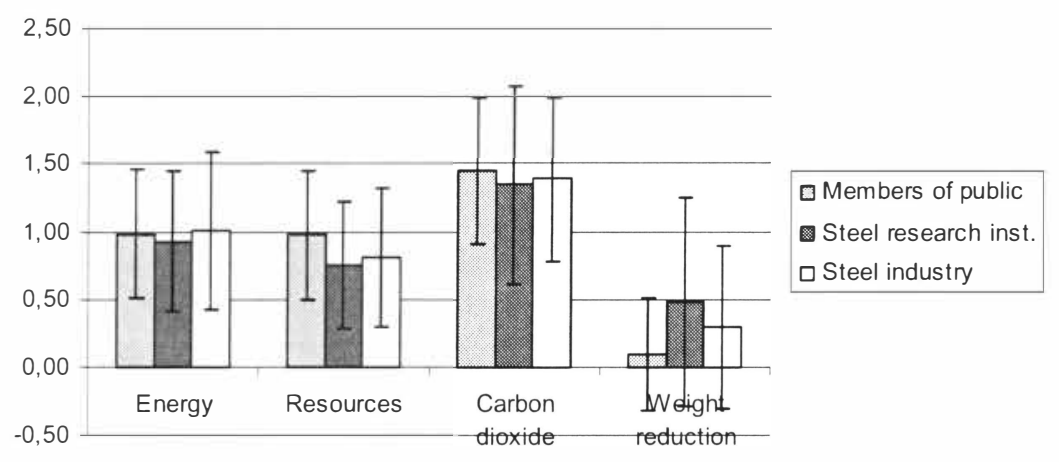

Figure 3. Regression coefficients (MLR) for respondents from the groups members of public, steel research institutes and steel industry, equalling one half of the factorial effects.

\section{RESULT AND DISCUSSION}

\subsection{Multiple linear regression}

Most methods of analysis of conjoint studies are made on a group level, with estimations assessed from average response. In Figure 3 the results from members of public, steel research institutes and steel industry, can be seen as examples of how the original groups of the study prioritize the environmental objectives of the research programme (and thus sustainability problems of the trade). The individual differences inflate the standard deviation to become as large as the average for each group, thus obstructing the evaluation. With this method of analysis, the segmentation among the respondents has to be based on a priori assumptions.

\subsection{Partial least squares regression (PLSR)}

PLSR enables the researcher to present the results individually and thereby the risk of misinterpretations of the result is decreased. The PLSR plot links the individual preferences to the design variables, thus the closer to a design variable that an individual response is found, the clearer the respondent has prioritised this design variable. In Figure 4 it is clear that most respondents focus on emissions of carbon dioxide, use of non-renewable energy and use of non-renewable resources. Weight reduction of products however is generally not an important issue (small influence on the model will put the attribute close to zero on both axes).

One of the research hypotheses was that the original six groups would separate from each other in the result. This was not the case, however, and from the PLSR-plot there was no clear segmentation into the six original groups. 


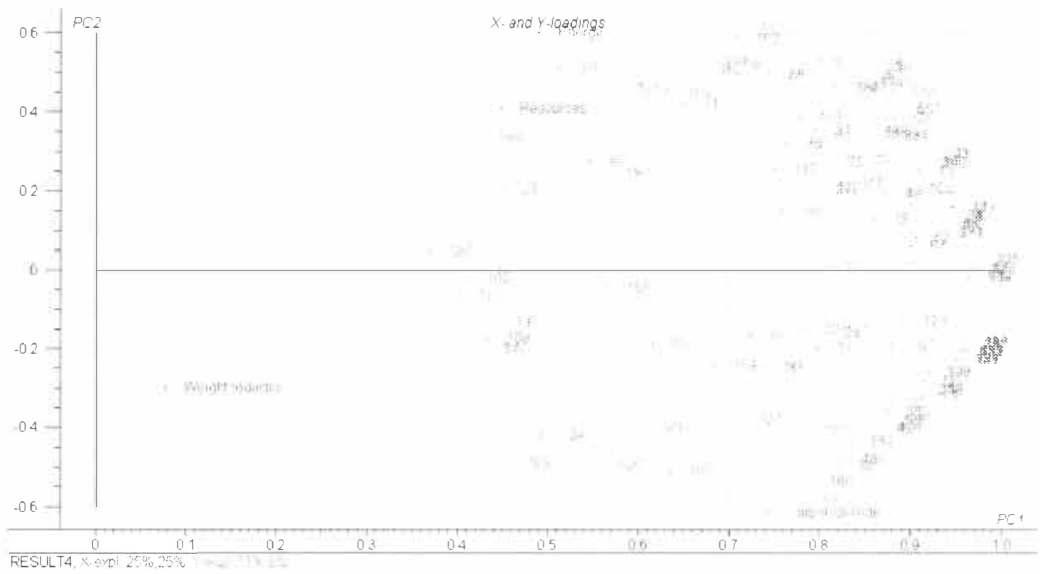

Figure 4. PLS2 regression loadings (preliminary results), linking individual preferences to the design variables.

\subsection{Cluster analysis}

In order to find any hidden segments a hierarchical cluster analysis was performed where the individual regression coefficients were used as classifiers. The preliminary results indicate eight segments with the following main preferences:

1. Carbon dioxide

2. Use of non-renewable resources/weight reduction

3. Use of non-renewable resources

4. Carbon dioxide, use of non-renewable energy and use of non-renewable resources

5. Use of non-renewable energy/carbon dioxide

6. Weight reduction

7. Carbon dioxide/use of non-renewable energy

8. Use of non-renewable energy/weight reduction

In Figure 5, segment 3 and 6 are shown as an example of how these new segments may be presented using MLR and in Figure 6, the same result is shown in a PLSR-plot.

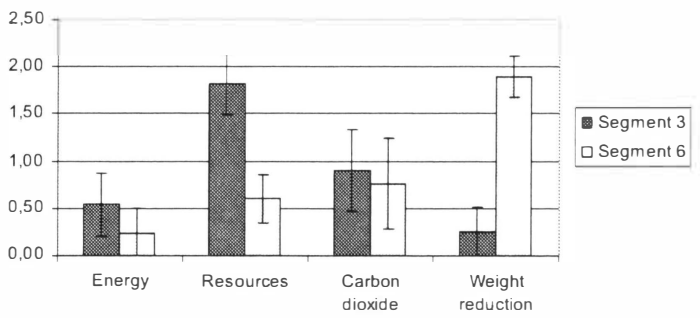

Figure 5. Average regression coefficients (MLR) with standard deviations for respondents from segments 3 and 6, equalling one half of the factorial effects. 


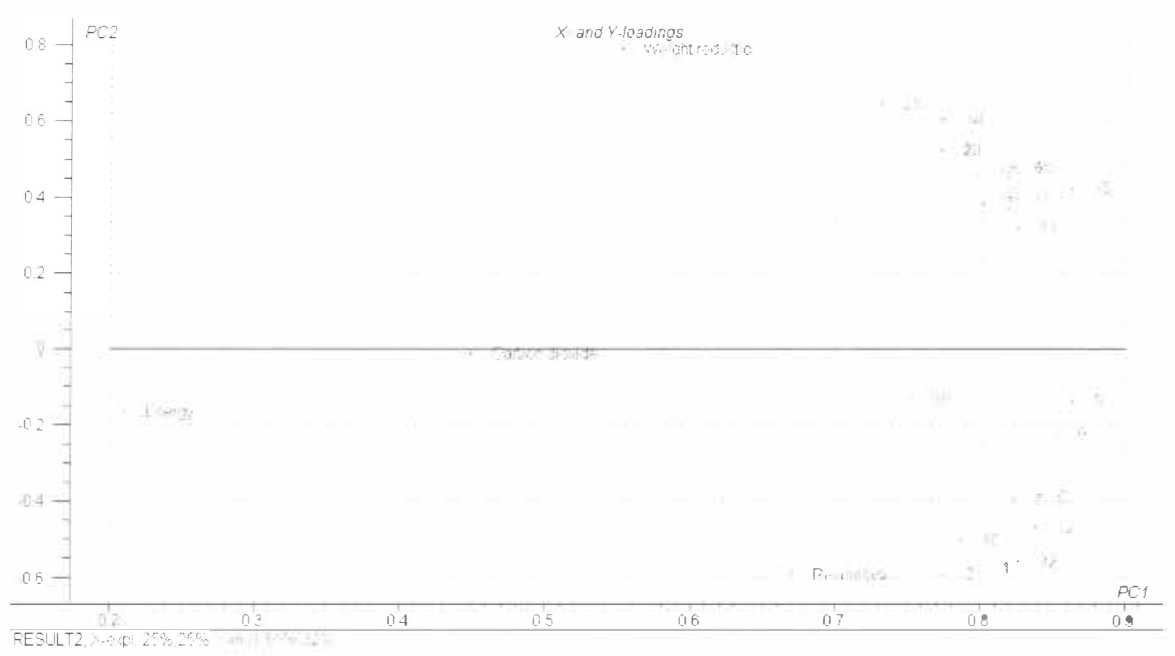

Figure 6. PLS2 regression loadings (preliminary results), linking individual preferences to the design variables for segment 3 and 6.

\subsection{Other important environmental concerns}

When the respondents were asked to chose the most important environmental issues from a list, use of natural resources, polluted air, polluted drinking water, climate change, loss of ozone layer and polluted ground water were all marked by more than half of the respondents. In comparison to the four assessed environmental targets there is a gap between the issues that the respondents chose if they are asked to choose freely, and the environmental objectives chosen by the Steel eco-cycle programme. People tend to find factors that influence themselves much more important than a factor that is abstract or vague.

\section{CONCLUSIONS}

From the four environmental objectives from the steel eco-cycle research programme, emission of carbon dioxide is prioritized by most respondents. More than half of the respondents have shown preference for carbon dioxide in one way or another. Use of nonrenewable resources and use of non-renewable energy are also important factors if the results are analyzed broadly. Weight reduction of products however, receives remarkably less attention.

The use of partial least squares regression in combination with cluster analysis has proved to be a good way of finding hidden segments within the respondents. The PLSR is also a superior method of presenting the results, since it enables the individual differences to be visualized.

When the result from the last part of the questionnaire is analyzed, it seems that people in general, as well as employees of the steel industry, prefers the industry to work with pollution control and hazardous substances rather than working with decreasing the use of non- 
renewable energy and non-renewable resources. The fact that so few of the respondents have chosen to prioritize weight reduction indicates that the industry has not successfully communicated the environmental benefits of these efforts.

Another main conclusion is that the environmental objectives set by the Swedish Steel Producers' Association, "Jernkontoret" for the research programme "Towards a closed steel eco-cycle", are not fully accepted and understood by the respondents. This communication issue also seems to exist within the trade and these results thus highlights the need for further research to develop an efficient environmental communication strategy.

\section{ACKNOWLEDGEMENTS}

This work was funded by the Foundation for Strategic Environmental Research (MISTRA) and the Swedish Steel Producers' Association (Jernkontoret) through the research programme "Towards a Closed Steel Ecocycle".

\section{REFERENCES}

[1] Alriksson, S. and T. Öberg, Conjoint analysis for environmental valuation - A review of methods and applications. Submitted, 2007.

[2] Herrmann, A., D. Schmidt-Gallas, and F. Huber, Adaptive conjoint analysis: understanding the methodology and assessing reliahility and validity, in Conjoint measurement - methods and applications, A. Gustafsson, A. Herrmann, and F. Huber, Editors. 2003, Springer Verlag. p. 305-329.

[3] Louviere, J.J., D.A. Hensher, and J.D. Swait, Conjoint preference elicitation methods in the broader context of random utility theory preference elicitation methods, in Conjoint measurement - methods and applications, A. Gustafsson, A. Herrman, and F. Huber, Editors. 2003, Springer Verlag. p. 331-370.

[4] Alriksson, S. and T. Öberg, Environmental risk perception - conjoint analysis to support strategic decisions and communication in the steel industry. Submitted, 2007.

[5] Moore, W.L. and M.B. Holbrook, Conjoint analysis on objects with environmentally. correlated attributes: the questionable importance of representative design. Journal of Consumer Research, 1990. 16: p. 490-497. 